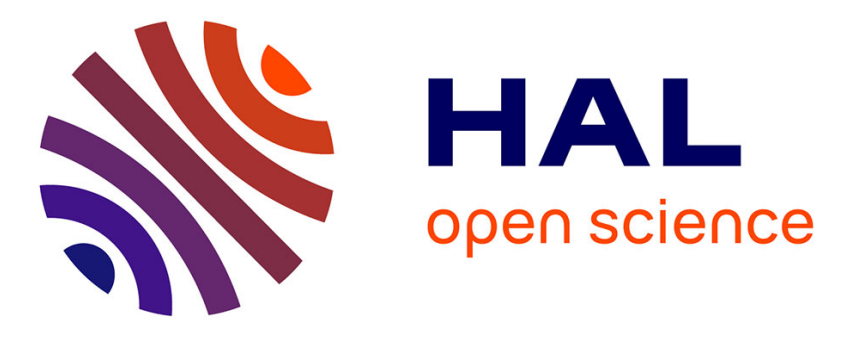

\title{
Optimal Transient Growth and Very Large-Scale Structures in Zero-Pressure Gradient Turbulent Boundary Layers
}

\author{
Grégory Pujals, Carlo Cossu, Sebastien Depardon
}

\section{To cite this version:}

Grégory Pujals, Carlo Cossu, Sebastien Depardon. Optimal Transient Growth and Very Large-Scale Structures in Zero-Pressure Gradient Turbulent Boundary Layers. 6th International Conference on Turbulence and Shear Flow Phenomena (TSFP-6), Jun 2009, Seoul, South Korea. hal-01053667

\section{HAL Id: hal-01053667 \\ https://hal-polytechnique.archives-ouvertes.fr/hal-01053667}

Submitted on 31 Jul 2014

HAL is a multi-disciplinary open access archive for the deposit and dissemination of scientific research documents, whether they are published or not. The documents may come from teaching and research institutions in France or abroad, or from public or private research centers.
L'archive ouverte pluridisciplinaire HAL, est destinée au dépôt et à la diffusion de documents scientifiques de niveau recherche, publiés ou non, émanant des établissements d'enseignement et de recherche français ou étrangers, des laboratoires publics ou privés. 


\title{
OPTIMAL TRANSIENT GROWTH AND VERY LARGE-SCALE STRUCTURES IN ZERO-PRESSURE GRADIENT TURBULENT BOUNDARY LAYERS
}

\author{
Gregory Pujals \\ LadHyX, \\ CNRS-École Polytechnique \\ F-91128 Palaiseau cedex France \\ gregory.pujals@ladhyx.polytechnique.fr \\ Carlo Cossu \\ LadHyX, \\ CNRS-École Polytechnique \\ F-91128 Palaiseau cedex France \\ carlo.cossu@ladhyx. polytechnique.fr
}

\author{
Sebastien Depardon \\ PSA Peugeot Citroën, \\ Centre Technique de Velizy, \\ 2 Route de Gisy, 78943 Vélizy-Villacoublay Cedex, France \\ sebastien.depardon@mpsa.com
}

\begin{abstract}
We are interested in the optimal energy growth of perturbations sustained by a zero pressure gradient turbulent boundary layer. We use the mean flow proposed by Monkewitz et al. (2007), the turbulence dynamics being modeled by an eddy viscosity added in the disturbance equations following the approach of del Álamo and Jiménez (2006), or Pujals et al. (2009) in the turbulent channel flow case. Although all the considered turbulent mean profiles are linearly stable, they support transient energy growths due to the non-normality of the operator. We find that the most amplified perturbations are streamwise uniform and correspond to streamwise vortices evolving into streamwise streaks.

Consistently with the study of del Álamo and Jiménez (2006), we find that two distinct peaks of the optimal growth exist for sufficiently large Reynolds numbers: a primary one scaling in outer units and a secondary one scaling in wall units. The optimal structures associated with the peak scaling in wall units correspond well to the most probable streaks observed in the buffer layer and their moderate energy growth is independent of the Reynolds number. The energy growth associated with the peak scaling in outer units is larger than that of the inner peak. The optimal perturbations associated with this primary peak consist in very large-scale structures with a spanwise wavelength of the order of $8 \delta$.

Since such very large-scale structures have not been observed yet in turbulent shear flows, preliminary experiments aiming at forcing such structures and studying their growth have been conducted. We find that large-scale turbulent streaks can be forced using well-shaped roughness elements embedded in the boundary layer. Their amplitude can reach about $13.5 \%$ of the free-stream velocity before decaying.
\end{abstract}

\section{INTRODUCTION}

Persistent streaky structures are commonly observed in turbulent shear flows. Many researches have been dedicated to the understanding of the mechanisms by which streaks are generated and of their relevance on the turbulent dynamics. In the near wall region of the boundary layer these streaks have a characteristic mean spanwise spacing of about $100 y^{+}$and are thought to play an essential role in a turbulent self-sustained mechanism. The 'lift-up' effect by which low energy streamwise vortices can induce large energy streaks is an important process embedded in this self-sustained mechanism. In the case of laminar shear flows, the same mechanism plays a crucial role in subcritical transition to turbulence (Reddy et al., 1998). In that case, the energy amplification of streaks, which is due to the non-normality of the linearized Navier-Stokes operator, is transient in time and proportional to the square of the Reynolds number. Thus, this amplification can lead to very large energy growths if optimized (Butler and Farrell, 1992; Schmid and Henningson, 2001).

Recent studies have also demonstrated that the well controlled optimal transient growth of artificially forced streaks can be efficiently used to manipulate at leading order laminar shear flows. Such a paradigm has been successfully applied to stabilize Tollmien-Schlichting waves in a laminar boundary layer (Cossu et al., 2002; Fransson et al., 2005) and to delay transition to turbulence (Fransson et al., 2006).

An extension of such approach consists in the manipulation of turbulent boundary layers with optimal or nearly optimal vortices and streaks, the first step being to compute the optimal perturbations of the turbulent boundary layer. We have therefore computed the optimal energy growth sustained by a zero pressure gradient turbulent boundary layer using the eddy viscosity associated with the turbulent mean velocity profile proposed by Monkewitz et al. (2007) in order to model the interaction between the turbulence dynamics and the perturbations. In a second part, we present the results of a preliminary experimental study in which very large-scale streaks are forced using spanwise organized 


\section{BACKGROUND}

\section{Turbulent mean flow}

The zero pressure gradient turbulent boundary layer mean flow $U(x, y), V(x, y)$, satisfies the mean mass and momentum conservation equations:

$$
\begin{aligned}
\frac{\partial U}{\partial x}+\frac{\partial V}{\partial y} & =0 \\
U \frac{\partial U}{\partial x}+V \frac{\partial U}{\partial y} & =\frac{1}{\rho} \frac{\partial \tau}{\partial y} .
\end{aligned}
$$

where $\tau=\nu(\partial U / \partial y)-\left\langle u^{\prime} v^{\prime}\right\rangle / \rho$ is the sum of the molecular viscosity and of the Reynolds shear stress. Here $x, y$ and $z$ are respectively the streamwise, normal and spanwise coordinates. At sufficiently large Reynolds numbers, the boundary layer mean velocity data can be fitted with asymptotic expressions. In the present study we use the self-consistent analytic expression recently proposed by Monkewitz et al. (2007) that fits very well the experimental data of Osterlund (1999) and Nagib et al. (2004) for a wide range of Reynolds numbers:

$$
\frac{U}{u_{\tau}}=\left[U_{i}^{+}\left(y^{+}\right)-U_{l o g}^{+}\left(y^{+}\right)+U_{e}^{+}\left(R e_{\delta^{*}}\right)-U_{w}^{+}(\eta)\right]
$$

where $u_{\tau}=\left(\nu d U /\left.d y\right|_{\text {wall }}\right)^{1 / 2}$ is the wall friction velocity, $y^{+}=y u_{\tau} / \nu$ is the wall normal coordinate scaled in inner units, $U_{e}^{+}=U_{e} / u_{\tau}$ is the free-stream velocity $U_{e}$ scaled with $u_{\tau}, R e_{\delta^{*}}=U_{e} \delta^{*} / \nu$ is the Reynolds number scaled on the displacement thickness $\delta^{*}$, and $\eta=y / \Delta$ is the wall normal coordinate scaled with the Rotta-Clauser length scale $\Delta=\delta^{*} U_{e}{ }^{+}$. The inner and outer coordinates satisfy $y^{+}=R e_{\delta *} \Delta$. The shear stress associated with the mean flow $U$ can be obtained by integrating eqn. (2) in the wall normal direction; the details of this integration are not reported here but are fully described in Cossu et al. (2009).

\section{Linearized equations and optimal growth}

Small perturbations $\mathbf{u}=(u, v, w), p$ to the turbulent mean flow $\mathbf{U}=(U(y), 0,0)$ satisfy the continuity $\nabla \cdot \mathbf{u}=0$ and the linearized momentum equation:

$$
\begin{gathered}
\frac{\partial \mathbf{u}}{\partial t}+U \frac{\partial \mathbf{u}}{\partial x}+(v \partial U / \partial y, 0,0)= \\
-\nabla p+\nabla \cdot\left[\nu_{T}(y)\left(\nabla \mathbf{u}+\nabla \mathbf{u}^{T}\right)\right]
\end{gathered}
$$

Due to the homogeneous nature of the mean flow in the streamwise and spanwise directions, we consider perturbations of the form $\mathbf{u}(x, y, z, t)=\widehat{\mathbf{u}}(\alpha, y, \beta, t) \mathrm{e}^{i(\alpha x+\beta z)}$, where $\alpha$ and $\beta$ are the streamwise and spanwise wavenumbers, respectively. Standard manipulations (see Schmid and Henningson, 2001), generalized to include a variable viscosity (Reynolds and Hussain, 1972), allow to rewrite the linearized system into the following generalized Orr-Sommerfeld and Squire equations for the normal velocity $\widehat{v}(y)$ and vorticity $\widehat{\omega_{y}}(y)$ :

$$
\begin{array}{r}
{\left[\begin{array}{cc}
D^{2}-k^{2} & 0 \\
0 & 1
\end{array}\right] \frac{\partial}{\partial t}\left\{\begin{array}{c}
\widehat{v} \\
\widehat{\omega_{y}}
\end{array}\right\}=} \\
{\left[\begin{array}{cc}
\mathcal{L}_{\mathcal{O S}} & 0 \\
-i \beta U^{\prime} & \mathcal{L}_{\mathcal{S} \mathcal{Q}}
\end{array}\right]\left\{\begin{array}{c}
\widehat{v} \\
\widehat{\omega_{y}}
\end{array}\right\}}
\end{array}
$$

$$
\begin{array}{r}
\mathcal{L}_{\mathcal{O S}}=-i \alpha\left[U\left(D^{2}-k^{2}\right)-U^{\prime \prime}\right]+ \\
\nu_{T}\left(D^{2}-k^{2}\right)^{2}+2 \nu_{T}^{\prime}\left(D^{3}-k^{2} D\right)+\nu_{T}^{\prime \prime}\left(D^{2}+k^{2}\right) \\
\mathcal{L}_{\mathcal{S} \mathcal{Q}}=-i \alpha U+\nu_{T}\left(D^{2}-k^{2}\right)+\nu_{T}^{\prime} D
\end{array}
$$

where $D$ and $\left({ }^{\prime}\right)$ stand for $\partial / \partial y$ and $k^{2}=\alpha^{2}+\beta^{2}$.

Even though the mean velocity profiles are linearly stable for all $\alpha$ and $\beta$, so that infinitesimal perturbations decay after enough time (Cossu et al., 2009), some perturbations may support large growth before decaying. The ratio $\|\widehat{\mathbf{u}}(t)\|^{2} /\left\|\widehat{\mathbf{u}}_{0}\right\|^{2}$, where \|\| stands for the energy norm, quantifies the energy amplification of a perturbation as it evolves in time. The temporal optimal growth $\widehat{G}(\alpha, \beta, t)=$ $\sup _{\widehat{\mathbf{u}}_{0}}\|\widehat{\mathbf{u}}(t)\|^{2} /\left\|\widehat{\mathbf{u}}_{0}\right\|^{2}$ is the maximum energy amplification of a disturbance optimized over all possible initial conditions $\widehat{\mathbf{u}}_{0}$. In this study, we focus on the maximum optimal growth $G_{\max }(\alpha, \beta)=\sup _{t} \widehat{G}(\alpha, \beta, t)$ reached using the optimal initial conditions.

\section{RESULTS}

\section{Optimal perturbations for a fixed Reynolds number}

First, the optimal perturbations and their growth are computed for a Reynolds number fixed to $\operatorname{Re}_{\delta^{*}}=17300$ and for a range of streamwise and spanwise wavenumbers extending from zero to $10^{4} / \Delta$. As in laminar shear flows or in turbulent Poiseuille flow, noticeable energy growths are obtained for streamwise elongated structures $(\alpha<\beta)$ while the maximum energy amplification is reached for streamwise uniform initial disturbances (i.e. $\alpha \Delta=0$ ) with a spanwise wavenumber $\beta \Delta \sim 3.7$ corresponding to $\lambda_{z} \approx 7.6 \delta$ (see Figure 1).

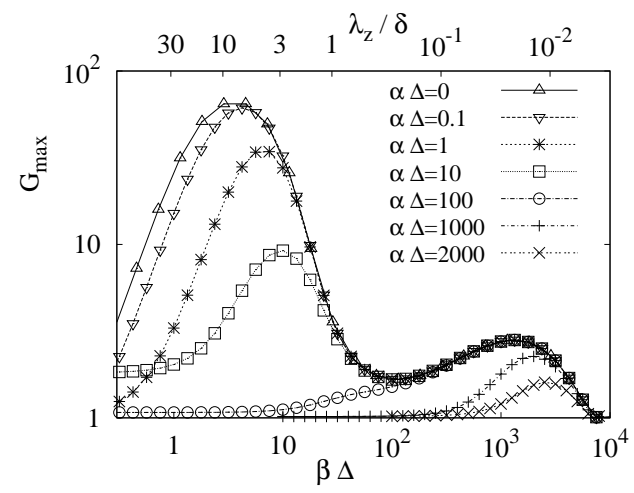

Figure 1: Maximum growth $G_{\max }$ as a function of the spanwise wavenumber $\beta \Delta$ obtained at $R e_{\delta^{*}}=17300$ for selected streamwise wavenumbers.

The double peak structure observed in turbulent channel flow case by del Álamo and Jiménez (2006) is also observed in the present case. A distinct secondary peak can be identified at large spanwise wavenumbers $\beta \Delta \sim 1300$ corresponding to $\lambda_{z}^{+} \sim 80$. The secondary peak becomes the only and dominant one for perturbations of large streamwise wavenumber $(\alpha \Delta>50)$.

We present in figure 2 the optimal initial conditions, along with their optimal responses, corresponding to the two peaks illustrated in figure 1. The initial disturbances (reported as a vector-map) consist in counter rotating streamwise vortices which induce at time of maximum amplification streamwise streaks (black and grey contours). The optimal perturbations associated with the secondary inner-scaling 
peak are plotted in Fig. 2(a). Both the initial vortices and the resulting streaks have their center around $y^{+} \sim 10$ and are confined to the buffer layer of the boundary layer. This structures correspond well to the most probable streaks commonly observed in turbulent shear flows (see Smith and Metzler, 1983). The optimal disturbances associated with the primary peak consist in very large-scale structures spreading the whole boundary layer, the optimal vortices being centered near the boundary layer edge.
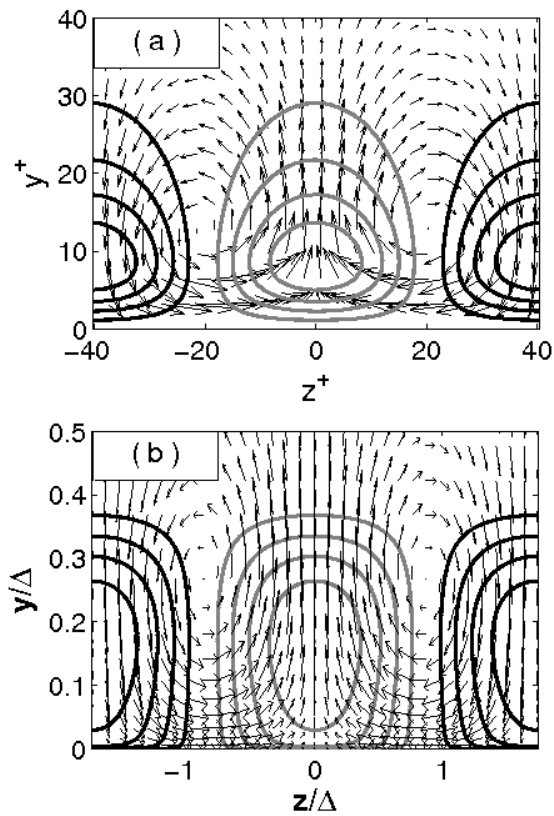

Figure 2: Cross-stream view of the $v-w$ component of the optimal initial vortices (arrows) and of the $u$ component of the corresponding maximally amplified streak (contourlines) for $\operatorname{Re}_{\delta^{*}}=17300, \alpha=0$. (a) the secondary peak optimal $(\beta \Delta=1300)$ is plotted in wall units while (b) the primary peak optimal $(\beta \Delta=3.65)$ is plotted in outer units. Black contours represent positive $u$ while grey contours represent negative $u$.

\section{Dependence on Reynolds number}

The computation of streamwise uniform optimals is repeated for a wide range of Reynolds numbers $R e_{\delta^{*}}$ from $10^{3}$ to $610^{4}$. Figure 3 presents the gains obtained varying $\beta \Delta$ in the range already investigated for the selected Reynolds numbers. It can be seen that both the outer and inner peaks are observable provided that the Reynolds number is large enough (as an indication for $R e_{\delta^{*}}>2000$ according to our computations). The secondary peak seems independent of the Reynolds number and is shifted towards larger values of $\beta \Delta$ as $R e_{\delta^{*}}$ increases. When replotted in wall units (not reported here, see Cossu et al., 2009), we find that this secondary peak is obtained for $\lambda_{z}=81.5 y^{+}$for all the considered Reynolds numbers. The maximum energy growth corresponding to the primary peak increases with the Reynolds number $R_{\delta^{*}}$ and is attained for spanwise wavenumbers in the range $\beta \Delta \in[1,10]\left(\lambda_{z} / \delta \in[3,20]\right)$, the maximum being reached for $\beta \Delta \approx 3$ corresponding to $\lambda_{z} / \delta \approx 8$.

\section{PRELIMINARY EXPERIMENTAL RESULTS}

\section{Description of the facility}

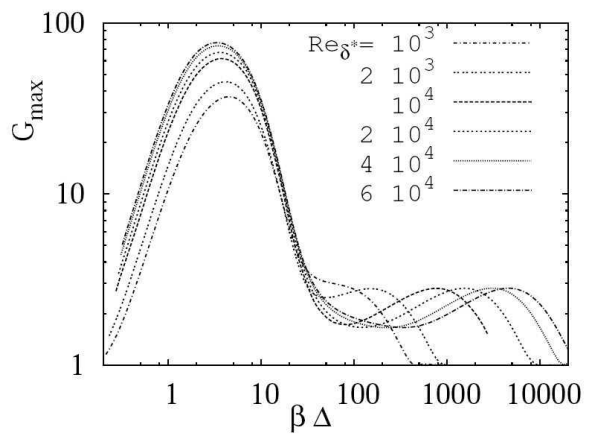

Figure 3: Maximum growth $G_{\max }$ of streamwise uniform $(\alpha=0)$ optimal perturbations as a function of the spanwise wavenumber for the selected Reynolds numbers $R e_{\delta^{*}}$.

A preliminary experimental validation of these theoretical results has been conducted in the wind-tunnel facility of the technical center of PSA Peugeot Citröen. The windtunnel is of closed-return type. The test section is $0.8 \mathrm{~m}$ long with a cross sectional area of $0.3 m \times 0.3 m$. The temperature can be kept constant and uniform within $\pm 0.5 C$. The contraction ratio is 8 and the velocity can be controlled from $7 m . s^{-1}$ up to $45 m . s^{-1}$. Due to the small dimensions of the test section, the developing boundary layer is quite thin: setting the free-stream velocity to $U_{e}=20 \mathrm{~m} . \mathrm{s}^{-1}$ the boundary layer is $\delta_{0}=5.4 \mathrm{~mm}$ thick at $x_{0}=110 \mathrm{~mm}$. The resulting Reynolds number $R e_{\delta^{*}}$ is $R e_{\delta^{*}} \approx 1000$ which is, according to our computations, just large enough to see the primary outer peak. The goal of this study is: (a) to see if artificially forced large-scale velocity streaks can be amplified in a turbulent boundary layer, (b) to study if the spanwise wavelength selected theoretically can be can be experimentally forced.

\section{Experimental setup}

To force the streaks in the boundary layer, we use cylindrical PVC roughness elements. The dimensions of these elements are of great importance if we want to generate stable streaks. The most relevant parameters are: the height of the cylinders $k$, their diameter $d$ and the spanwise spacing $\lambda_{z}$. In this study, we keep the height of the roughness elements constant equal to $k=4 m m(k / \delta=0.8)$, as well as the ratio $d / \lambda_{z}=4$ (the same ratio was used in Fransson et al. $(2005,2006)$; Holland and Cossu (2009)). Several wavelength spanning the width of the primary peak discussed above are tested. The selected wavelength are nearby $\lambda_{z} / \delta_{0}=3,5,6,7.5,10$ and 12 . The figure 4 is a picture of one configuration, the cylinders span at least $2 / 3$ of the test section. They are pasted on a thin scotch tape $(<0.1 \mathrm{~mm}$ thick) and painted in black to limit reflections due to the laser sheet.

The velocity is measured using Dantec's Flow Manager particle image velocimetry (PIV) system associated with a $10 \mathrm{MW}$ Yag laser and a $1024 \times 1280$ Hisense Mk2 CCD camera placed above the test section (resulting in $(x, z)$ planes). A $28 \mathrm{~mm}$ optical lens is used resulting in $300 \times 220 \mathrm{~mm}^{2}$ field of view. The laser sheet is $1 \mathrm{~mm}$ thick and, in order to ensure the convergence of the mean velocity fields, 600 pairs of images are acquired. All the data presented here are acquired at $Y=2 m m(Y=k / 2)$ from the wall.

Different measures of the streaks amplitude are often used in literature such as the kinetic energy or the min-max definition used in Fransson et al. (2005). However, because 


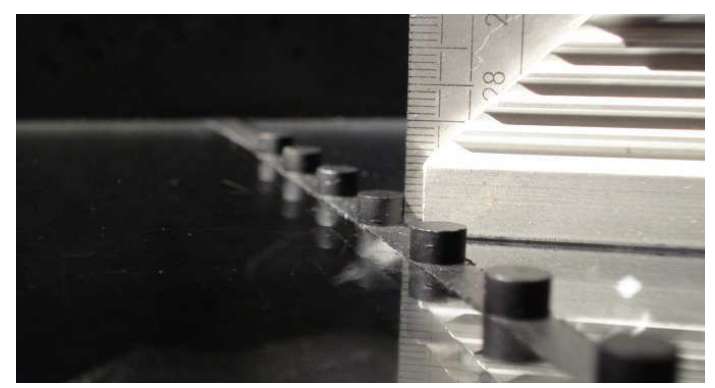

Figure 4: Visualization of the experimental setup for $\lambda_{z}=$ $5 \delta_{0}$. The $4 \mathrm{~mm}$ high cylindrical roughness elements are pasted on the test section using a thin scotch tape.

this apparatus does not allow us to quantify the kinetic energy contained in the vortices induced by the roughness elements, we use a measure of the amplitude of the induced streaks based on a fit of the streamwise velocity to a sinusoidal function defined as $\widehat{u}(x, Y, z)=\bar{u}(x, Y, z)+$ $\widehat{A}(x, Y) \sin \left[2 \pi\left(z-z_{0}\right) / \lambda_{z}\right]$ (see Holland and Cossu, 2009). An approximation from below of the amplitude of the streaks as a function of the streamwise distance can be retrieved through the relation $\widehat{A_{s t}}(x, Y)=\widehat{A}(x, Y) / 2 U_{e}$.

\section{Experimental large-scale streaks}

Figure 5 shows a visualization of the mean flow measured downstream of the $\lambda_{z}=6 \delta_{0}$ cylinder array. The flow is from left to right and the cylinders (not represented) are located at $z / \lambda_{z}= \pm 0.5, \pm 1.5$ and \pm 2.5 on the left side of the figure. The streamwise velocity scaled on the free-stream velocity $U_{e}$ is plotted versus the streamwise and spanwise directions both scaled on the wavelength $\lambda_{z}$. Due to the presence of the roughness elements, the mean flow is clearly spanwise modulated and an alternating pattern of high speed (clear contours) and low speed (dark contours) streaks is observed. In accordance with Fransson et al. (2005), it appears that the high speed streaks are developing straight behind the cylinders.

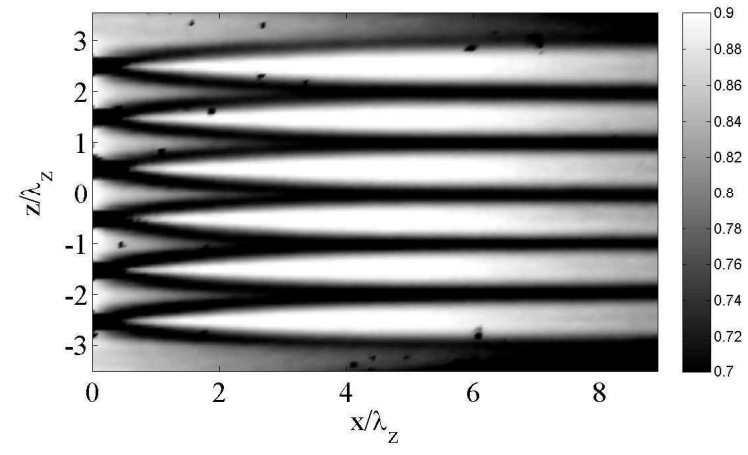

Figure 5: Very large-scale coherent streaks forced in a turbulent boundary layer in the plane situated at $Y=k / 2$ from the wall. The spanwise wavelength used here is $\lambda_{z} \approx 6 \delta_{0}$. Average of the streamwise velocity component scaled on the free-stream velocity $U / U_{e}$ measured using PIV.

In figure 6 , we present the normalized spanwise mean velocity distribution $U\left(x=3.7 \lambda_{z}, z / \lambda_{z}\right) / U_{e}$ as a function the spanwise direction $z / \lambda_{z}$ (open circles). This velocity profile is very close to a sinusoidal profile. The corresponding best sinusoidal fit (see Holland and Cossu, 2009) is also displayed in the solid line. The agreement between the two curves is fairly good taking into account the possible scatter in experimental data due to the laser sheet thickness and the velocity gradient in the near wall region. The amplitude $\widehat{A_{s t}}(x, Y)$ issuing from the sinusoidal fit is therefore a reasonable estimation of the streaks finite amplitude.

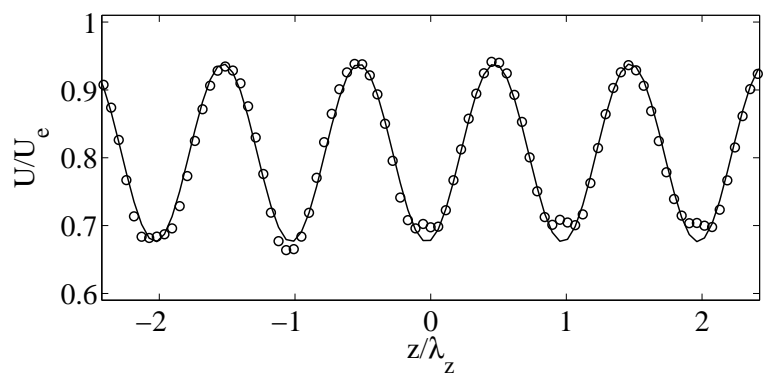

Figure 6: Streamwise velocity profile $U / U_{e}$ (open circles) and its best sinusoidal fit (solid line) plotted versus $z / \lambda_{z}$ at $x / \lambda_{z} \approx 3.7$.

In figure 7 , the amplitude of the velocity streaks is plotted as a function of the streamwise distance scaled by the spanwise spacing between the roughness elements. Despite the fact that the cylinders used here are slightly lower than the boundary layer thickness, the amplitude of the forced streaks reaches about $13.5 \%$ of the free-stream velocity at a distance $x_{\max } \approx 4 \lambda_{z}$ before decaying further downstream. Results obtained with other wavelengths lead to similar conclusions, very large-scale turbulent velocity streaks can be forced and are amplified, the streamwise distance over which the maximum amplitude is attained scales with the wavelength $\lambda_{z}: x_{\max } \approx 4 \lambda_{z}$.

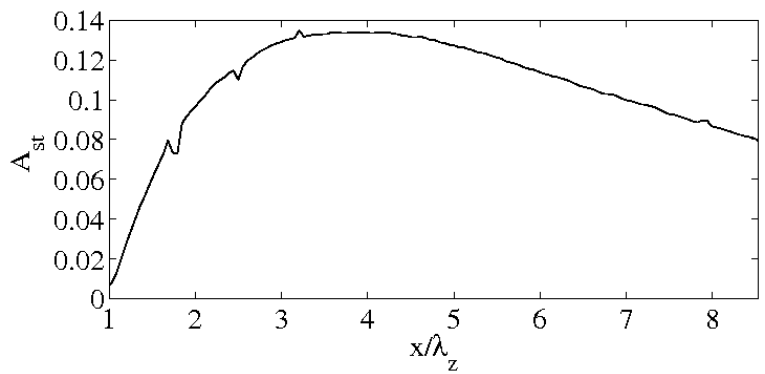

Figure 7: Streaks amplitude $\widehat{A_{s t}}(x, Y)$ as a function of the streamwise coordinate scaled with the wavelength $x / \lambda z$.

Even though, the mean flows involved here are different (the analytic velocity profile proposed by Monkewitz et al. (2007) being valid for high Reynolds numbers), the relative amplitudes for each tested wavelength are in good agreement with theoretical predictions. In Fig. 8 we have reported the normalized amplification curve obtained using the analytic profile (solid line) along with the experimental results (closed circles) normalized with the maximum amplitude $\widehat{A_{s t}}$. The global maximum amplitude $\max \left(\widehat{A_{s t}}\right)$ is obtained around $\lambda_{z}=6 \delta_{0}$ while larger and smaller wavelengths streaks reach a lower amplitude.

\section{SUMMARY AND DISCUSSION}

We have computed the linear optimal energy growth sustained by a zero pressure gradient turbulent boundary layer. The mean velocity profile used here is the two scale composite expansion proposed by Monkewitz et al. (2007). It is found that 


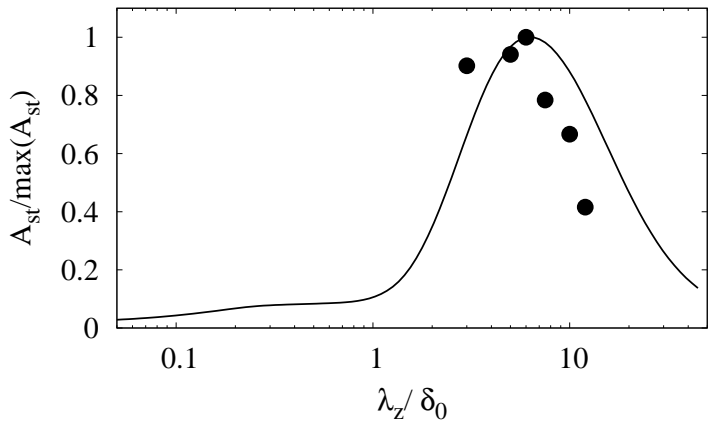

Figure 8: Experimental (closed circles) amplitude normalized with the maximum value, as a function of the spanwise wavelength scaled on the boundary layer thickness $\delta_{0}$ for $R e_{\delta^{*}}=1000$. For comparison, we plot in solid line the theoretical curve obtained with the analytic velocity profile at the same Reynolds number

- all the considered turbulent boundary layers are linearly stables,

- only streamwise elongated disturbances can be transiently amplified, the most amplified being streamwise uniform and consist in streamwise vortices leading to streamwise streaks,

- two distinct peaks of energy growth are found provided that the Reynolds number is large enough,

- the secondary peak does not depend on $R e_{\delta^{*}}$ and is attained for vortices and streaks with a spanwise spacing of $\lambda_{z}^{+}=81.5 y^{+}$,

- the energy amplification associated with the primary peak the largest one and the corresponding structures are very large-scale vortices with $\lambda_{z} \sim 8 \delta$ centered near the edge of the boundary layer while the amplified streaks fill the whole boundary layer.

The streamwise streaks associated with the inner peak can be related to the most probable streaks observed in the buffer layer of turbulent shear flows.

In some recent studies, evidences of very large-scale streaks present in the outer region of turbulent shear flows (see Hutchins and Marusic, 2007; del Álamo and Jiménez, 2003) have been revealed. These streaks, being of finite streamwise extension, have a typical spanwise wavelength $\lambda_{z} \sim \delta$. However, to our best knowledge, there is no experimental or numerical observation of large-scale structures with $\lambda_{z} \approx 4 \sim 8 \delta$. In the core region of turbulent Couette flow, large-scale streamwise vortices and streaks are kown to exist for a long time (Komminaho et al., 1996). In a recent experimental study, Kitoh and Umeki (2008) confirmed that these very large-scale streaks can be artificially forced in turbulent Couette flow.

Following this approach and the experiments conducted by Fransson et al. $(2005,2006)$, we use an array of cylindrical roughness elements to force such structures in a turbulent boundary layer. Very large-scale structures with a spanwise spacing $3 \delta_{0}<\lambda_{z}<12 \delta_{0}$ can be forced and are amplified through the lift-up effect. The maximum amplitude obtained here is near $13.5 \%$ of the free-stream velocity for structures with $\lambda_{z}=6 \delta_{0}$.

\section{REFERENCES}

del Álamo, J.C. and Jiménez, J., 2003, "Spectra of the very large anisotropic scales in turbulent channels," Phys. Fluids Vol. 15, L41.

del Álamo, J. C. and Jiménez, J., 2006, "Linear energy amplification in turbulent channels," J. Fluid Mech. Vol. 559, pp. 205-213.

Butler, K. M. and Farrell, B. F., 1992, "ThreeDimensional Optimal Perturbations in Viscous Shear Flow," Phys. Fluids A Vol. 4, pp. 1637-1650.

Cossu, C. and Brandt, L., 2002, "Stabilization of Tollmien-Schlichting waves by finite amplitude optimal streaks in the Blasius boundary layer," Phys. Fluids Vol. 14, pp. L57-L60.

Cossu, C., Pujals, G. and Depardon, S., 2009, "Optimal transient growth and very large-scale structures in turbulent boundary layers," J. Fluid Mech. Vol. 619, pp. 79-94.

Fransson, J., Brandt, L., Talamelli, A. and Cossu, C., 2005, "Experimental study of the stabilisation of TollmienSchlichting waves by finite amplitude streaks," Phys. Fluids Vol. 17, pp. 054110-054125.

Fransson, J., Talamelli, A., Brandt, L. and Cossu, C., 2006, "Delaying Transition to Turbulence by a Passive Mechanism," Phys. Rev. Lett. Vol. 96, pp. 064501.

Holland, M. and Cossu, C., 2009, "Adding streaks in the plane Poiseuille flow," Comptes-Rendus de l'Acadmie des Sciences, Mcanique (in press).

Hutchins, N. and Marusic, I., 2007, "Evidence of very long meandering features in the logarithmic region of turbulent boundary layers," J. Fluid Mech. Vol. 579, pp. 128.

Kitoh, O., and Umeki, M., 2008, "Experimental study on large-scale streak structure in the core region of turbulent plane Couette flow," Phys. Fluids Vol. 20, pp.025107025118

Komminaho, J., Lundbladh, A. and Johansson, A. V., 1996, "Very large structures in plane turbulent couette flow," J. Fluid Mech. Vol. 320, pp. 259285.

Monkewitz, P. A., Chauhan, K. A. and Nagib, H. M., 2007, "Self-consistent high-reynolds number asymptotics for zero-pressure-gradient turbulent boundary layers," Phys. Fluids Vol. 19, pp. 115101.

Nagib, H. M., Christophorou, C. and Monkewitz, P. A., 2004, "High Reynolds Number Turbulent Boundary Layers Subjected to Various Pressure-Gradient Conditions," In IUTAM Symposium on One Hundered Years of Boundary Layer Research, ed. G. Meier and K. Sreenivasan, Göttingen, Germany: Springer, pp. 383-394. DLR.

Osterlund, J. M., 1999, "Experimental studies of zeropressure gradient tubulent boundary layer flows," $\mathrm{PhD}$ thesis, Royal Insitute of Technology (KTH), Stockholm.

Pujals, G., Garcìa-Villalba, M., Cossu, C. and Depardon, S. 2009, "A note on optimal transient growth in turbulent channel flows," Phys. Fluids Vol. 21, pp. 015109-015115.

Reddy, S. C., Schmid, P. J., Baggett, J. S. and Henningson, D. S., 1998, "On the stability of streamwise streaks and transition thresholds in plane channel flows," J. Fluid Mech. Vol. 365, pp. 269-303.

Reynolds, W. C. and Hussain, A. K. M. F., 1972, "The mechanics of an organized wave in turbulent shear flow. Part 3. Theoretical models and comparisons with experiments," J. Fluid Mech. Vol. 54, pp. 263-288.

Smith, J. R. and Metzler, S. P., 1983, "The characteristics of low-speed streaks in the near-wall region of a turbulent boundary layer," J. Fluid Mech. Vol. 129, pp. $27-54$.

Schmid, P. J. and Henningson, D. S., 2001, "Stability and Transition in Shear Flows," Springer, New York . 